OPEN ACCESS

Edited by:

Ruowen Zhang,

Southern Research Institute,

United States

Reviewed by:

Liu Hong,

Fourth Military Medical University,

China

Caiping Ren,

Central South University, China

*Correspondence:

Xing Huang

huangxing66@zju.edu.cn

0000-0002-8886-2777

${ }^{t}$ These authors have contributed

equally to this work

Specialty section:

This article was submitted to Molecular and Cellular Oncology, a section of the journal Frontiers in Cell and Developmental Biology

Received: 03 September 2020

Accepted: 19 October 2020

Published: 12 November 2020

Citation:

Huang X and Zhang G (2020)

Split Cyclin-Dependent Kinase

4/6-Retinoblastoma 1 Axis

in Pancreatic Cancer.

Front. Cell Dev. Biol. 8:602352.

doi: 10.3389/fcell.2020.602352

\section{Split Cyclin-Dependent Kinase 4/6-Retinoblastoma 1 Axis in Pancreatic Cancer}

\author{
Xing Huang ${ }^{\star \dagger}$ and Gang Zhang ${ }^{\dagger}$ \\ Zhejiang Provincial Key Laboratory of Pancreatic Disease, School of Medicine, Zhejiang University, Hangzhou, China
}

Drugs targeting the cyclin-dependent kinase 4/6 (CDK4/6)-retinoblastoma 1 (RB1) axis have shown efficacy against multiple solid cancers, but their therapeutic potential in pancreatic cancer remains poorly defined. A recent report proposed that a "tailored" combination of first-line and second-line CDK4-targeting drugs would hold promise for pancreatic cancer treatment. Indeed, this therapeutic strategy exhibited significantly suppressive effects on pancreatic cancer patient-derived cell lines and tumor tissue in vitro. However, the study neglected immune involvement and the influence of CDK6 and RB1 in CDK4 inhibition-based treatment. Herein, we reveal multiple new facets of the CDK4/6-RB1 axis in pancreatic cancer, highlighting the complexity of this signaling axis for future prognostic and therapeutic targeting.

Keywords: CDK4/6, combination therapy, pancreatic cancer, precise treatment, prognosis, Rb1

\section{INTRODUCTION}

Pancreatic ductal adenocarcinoma (PDA) is the most prevalent pathohistological type of pancreatic cancer and is characterized by high lethality rates (Kleeff et al., 2016). Mutations in multiple tumor suppressor genes contribute to the tumorigenesis and progression of PDA, including but not limited to CDKN2A (Collisson et al., 2019). As a Polycomb repressed cell cycle checkpoint gene, CDKN2A encodes P16 $6^{\mathrm{INK} 4 \mathrm{~A}}$ that binds to cyclin D-cyclin-dependent kinase 4/6 (CDK4/6) to prevent retinoblastoma 1 (RB1) phosphorylation (Rubin, 2013). In its hypo-phosphorylated state, RB1 inhibits cell cycle progression through binding and inhibiting the E2F family of transcription factors (van den Heuvel and Dyson, 2008). Mutations in CDKN2A result in sustained RB1 phosphorylation, dysregulated cell cycle progression, and tumor cell proliferation. Recently, Chou et al. (2018) reported a "tailored" combination of first-line and second-line CDK4-targeting drugs for PDA therapy, guided by the predictive marker RB1. Although this strategy showed translational potential, genomic analyses suggest that the conventional CDK4/6-RB1 signaling pathway is divergent in pancreatic cancer, questioning the efficacy of this therapy.

Abbreviations: PAAD, pancreatic adenocarcinoma; PDA, pancreatic ductal adenocarcinoma; RB1, retinoblastoma 1; TCGA, The Cancer Genome Atlas. 
A

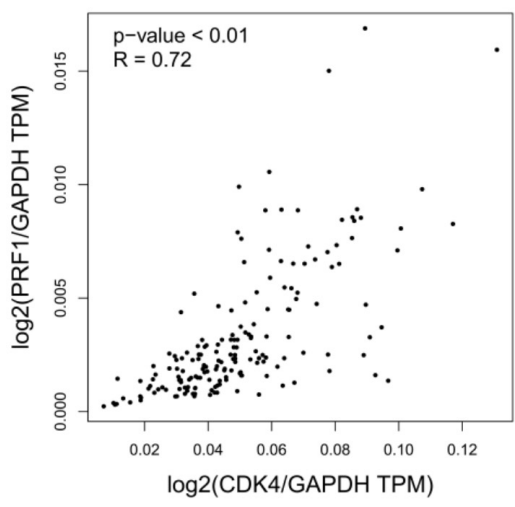

D

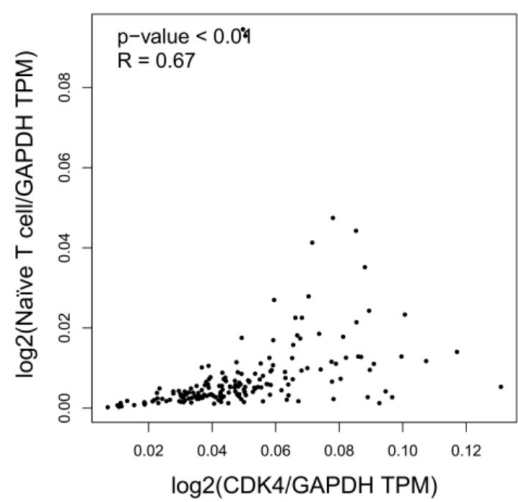

G

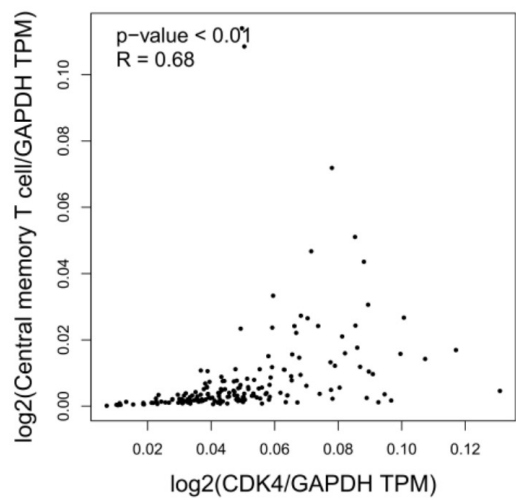

B

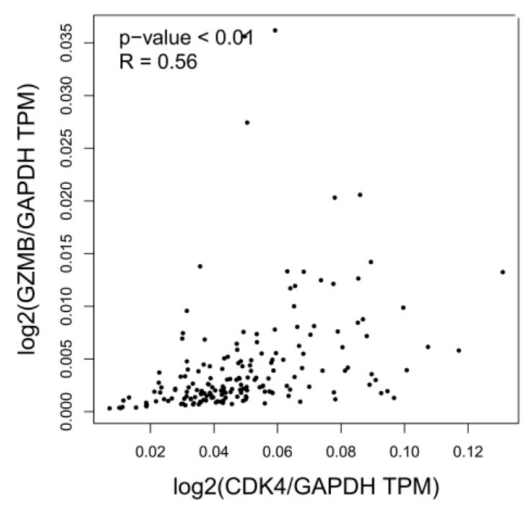

$\mathrm{E}$

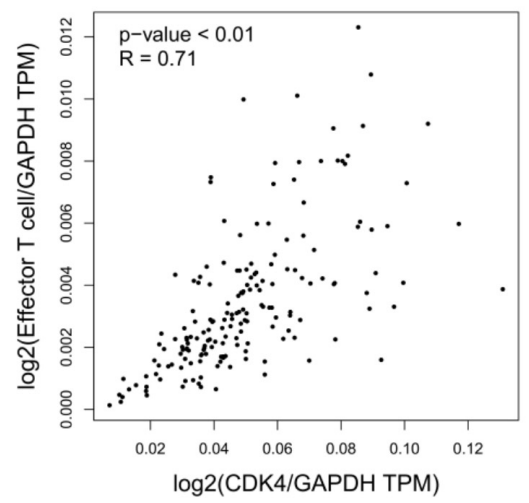

H

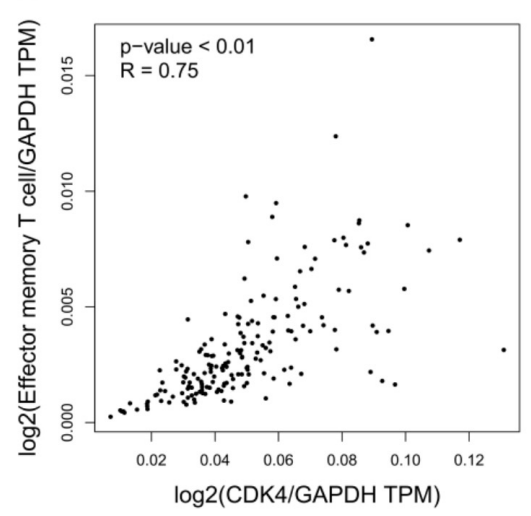

C

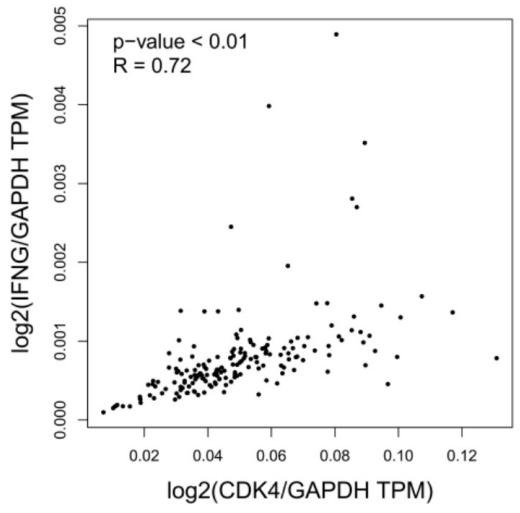

$\mathbf{F}$
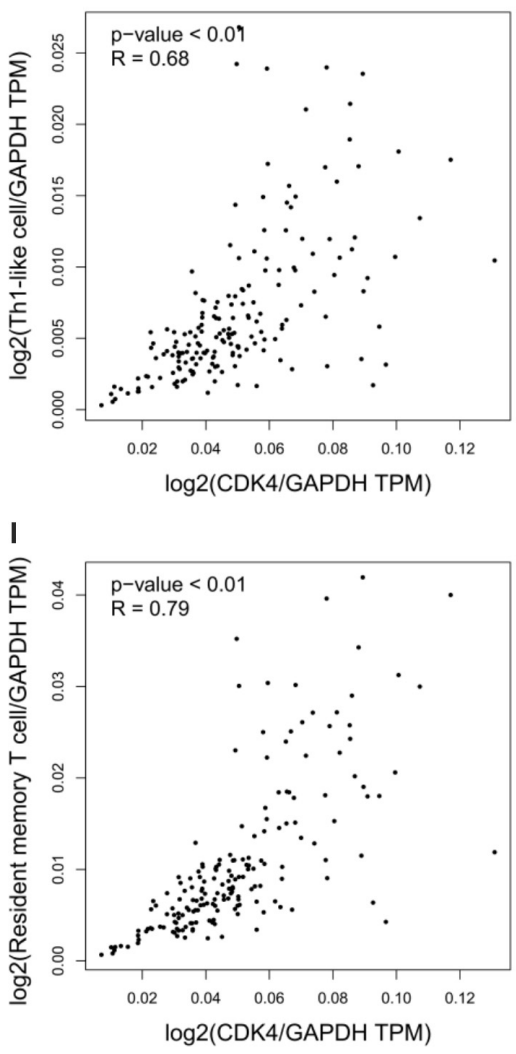

FIGURE 1 | CDK4 correlates with immune regulation in pancreatic cancer. (A-C) Correlation analysis of CDK4 and the immune effector molecules PRF1 (A), GZMB (B), and IFNG (C) in PAAD. (D-I) Correlation analysis of CDK4 and immune cell signatures in PAAD, including naïve T cell signatures (D), effector T cell signatures

(E), Th1-like cell signatures (F), central memory $T$ cell signatures (G), effector memory $T$ cell signatures $(\mathbf{H})$, and resident memory $T$ cell signatures (I).

$P$-values $\leq 0.05$ were considered statistically significant.

\section{METHODS}

Data on the prognostic potential of CDK4/6 and RB1 in pancreatic cancer were collected from The Cancer Genome Atlas $(\mathrm{TCGA})^{1}$ database. The Tumor and Immune System Interaction Database $^{2}$ was used to examine the correlation between

${ }^{1}$ http://cancergenome.nih.gov

${ }^{2}$ http://cis.hku.hk/TISIDB the abundance of immunomodulators and the expression of investigative genes. Gene Expression Profiling Interactive Analysis $2^{3}$ was used to calculate the prognostic index, including gene expression level and its correlation to patient survival. cBioPortal for Cancer Genomics ${ }^{4}$ was used to visualize and compare gene alterations. A one-way ANOVA was used to

\footnotetext{
${ }^{3} \mathrm{http} / / /$ gepia2.cancer-pku.cn

${ }^{4} \mathrm{http}: / /$ www.cbioportal.org
} 
A

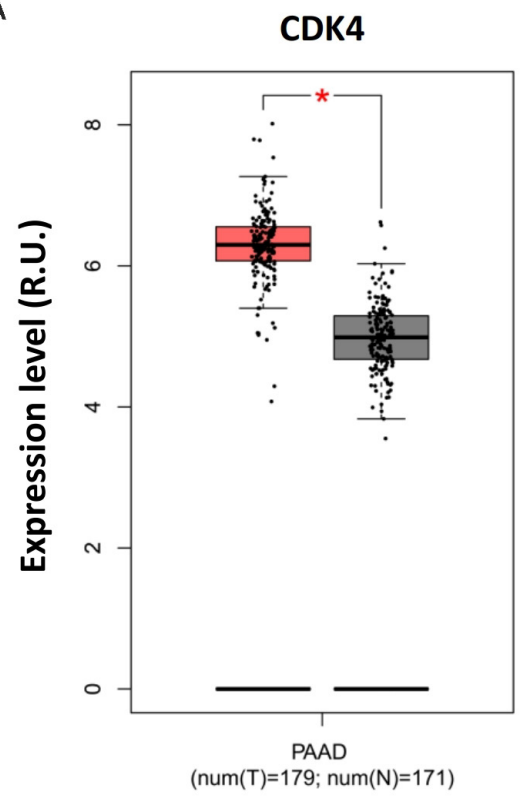

B

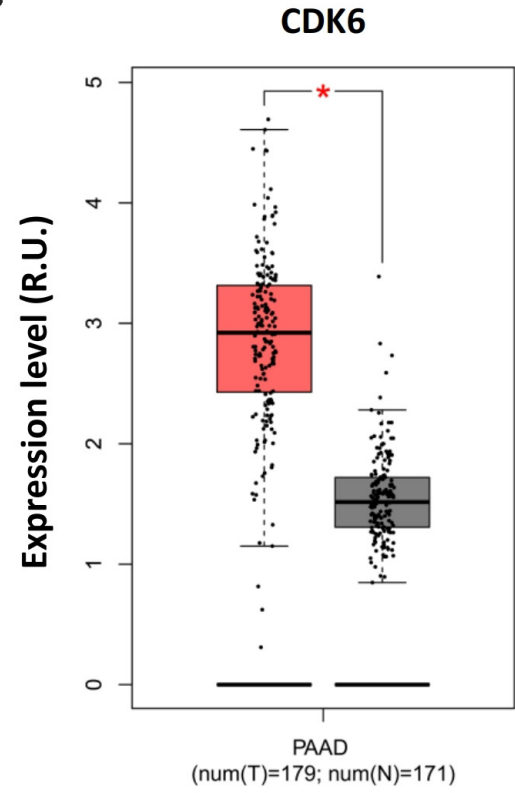

C

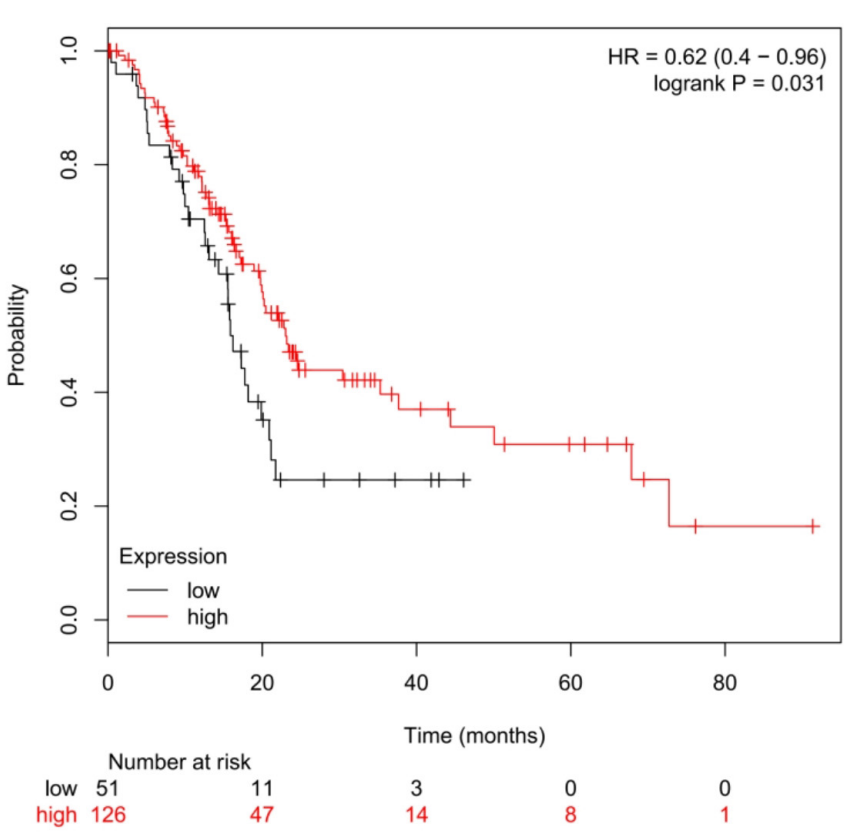

D

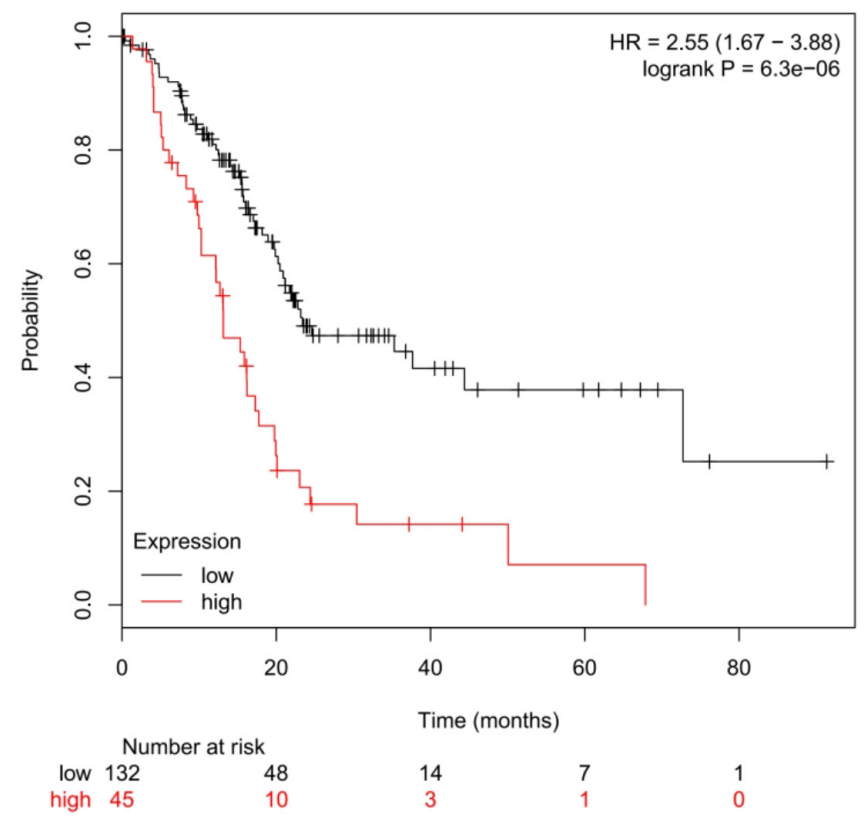

FIGURE 2 | Opposite prognoses of CDK4 and CDK6 in pancreatic cancer. Differential expression analysis of (A) CDK4 and (B) CDK6 in PAAD. OS analysis of (C) CDK4 and (D) CDK4 in PDA. P-values $\leq 0.05$ were considered statistically significant. ${ }^{*} p \leq 0.01$.

analyze the differential expression of CDK4/6 and RB1 and the abundance of immunomodulators. Genes with $|\log 2 \mathrm{FC}|$ values $>1$ and $q$ values $<0.01$ were considered statistically significant. Overall survival (OS) analyses of CDK4/6 and RB1 were assessed using the Kaplan-Meier method, with a $50 \%$ (median) cutoff for both low and high expression groups. The log-rank test (the Mantel-Cox test) was used for hypothesis testing, and the Cox proportional hazard regression model was used to calculate the hazard ratio. $P$-values $\leq 0.05$ were used as a threshold when ranking the data. Spearman correlation analysis was used to analyze the correlation between the pair-wise gene expression of CDK4 and a range 


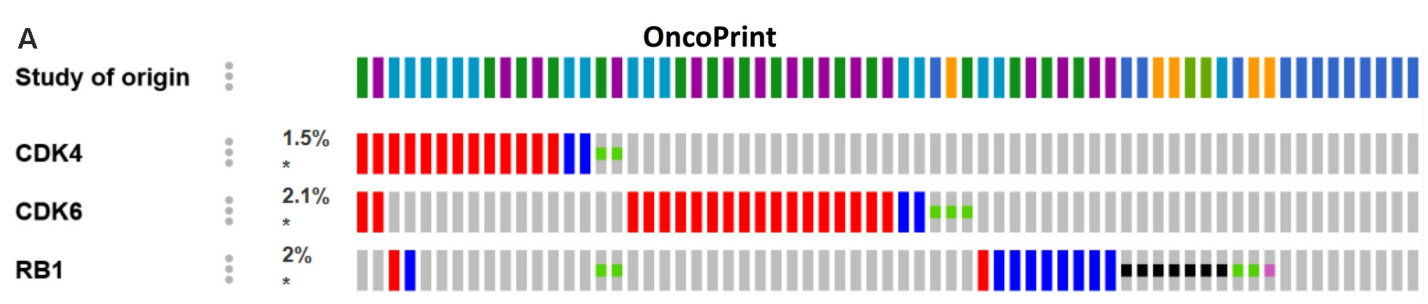

Missense Mutation (unknown significance)

Other Mutation

- Truncating Mutation (putative driver)

Amplification

Deep Deletion

No alterations

- Not profiled

Acinar Cell Carcinoma of the Pancreas (Johns Hopkins, J Pathol 2014)

Cystic Tumor of the Pancreas (Johns Hopkins, PNAS 2011)

Insulinoma (Shanghai, Nat Commun 2013)

Pancreatic Adenocarcinoma (ICGC, Nature 2012)

Pancreatic Adenocarcinoma (QCMG, Nature 2016)

IPancreatic Adenocarcinoma (TCGA, PanCancer Atlas)

Pancreatic Adenocarcinoma (TCGA, Provisional)

Pancreatic Cancer (UTSW, Nat Commun 2015)

| Pancreatic Neuroendocrine Tumors (Johns Hopkins University, Science 2011)

Whole-Genome Sequencing of Pancreatic Neuroendocrine Tumors (Nature, 2017)

B
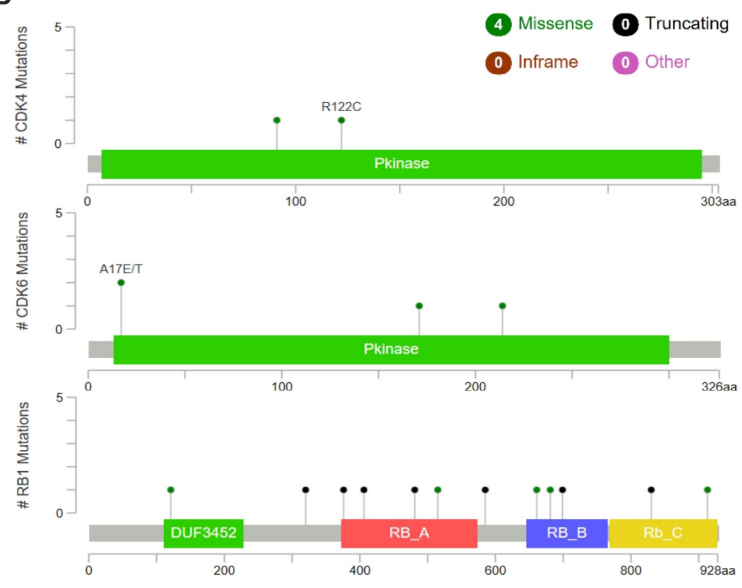

D

E
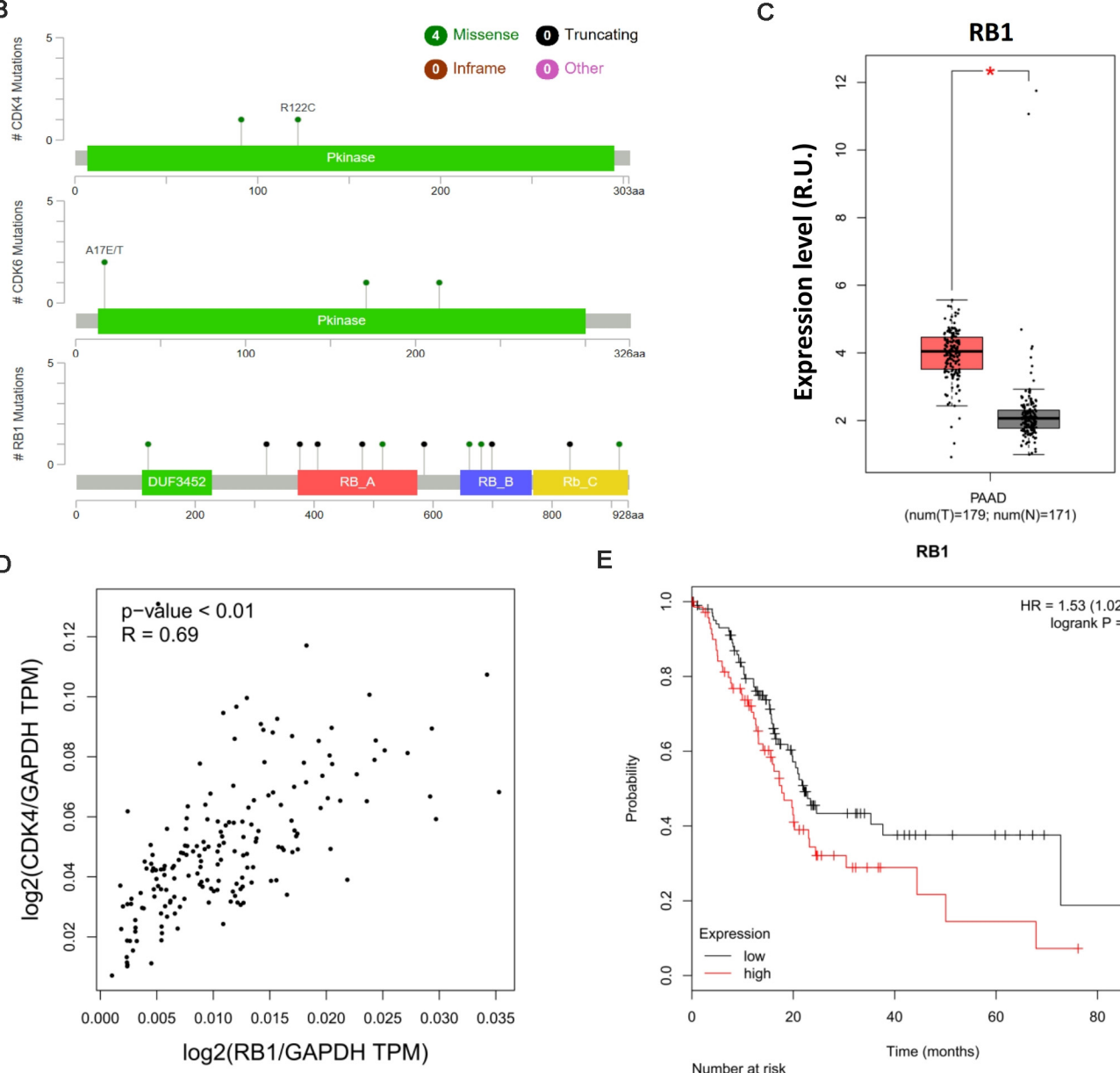

RB1

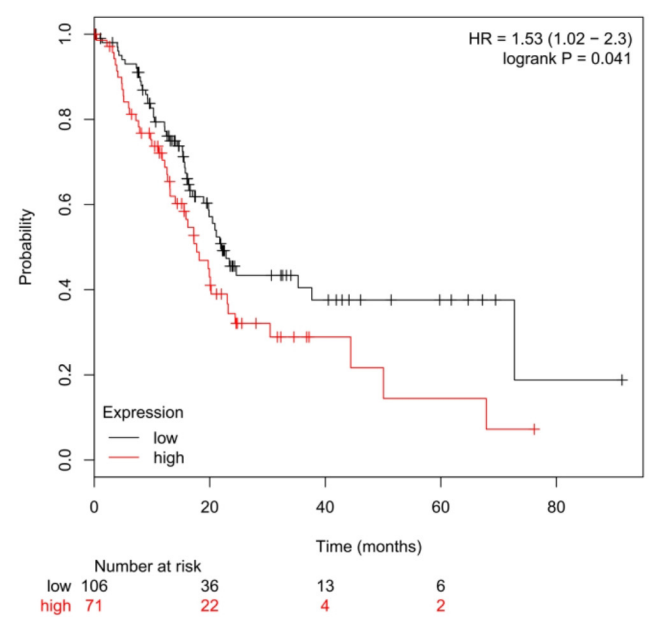

FIGURE 3 | RB1 couples with CDK4 and is related to poor prognosis in pancreatic cancer. (A) Landscape of the CDK4/6-RB1 axis in pancreatic cancer. Compact visualization of cases with different genetic alterations, including fusions, amplification, deletions, truncating mutations, and missense mutations. (B) Detailed mutational analysis of CDK4, CDK6, and RB1 in pancreatic cancer. (C) Differential expression analysis of RB1 in PAAD. (D) Correlation analysis of RB1 and CDK4 in PAAD. (E) OS analysis of RB1 in PAAD. P-values $\leq 0.05$ were considered statistically significant. ${ }^{*} p \leq 0.01$. 
of immunomodulators. $P$-values $<0.01$ were considered as significant. CDK4-related immunomodulators were collected, according to Charoentong et al.

\section{RESULTS}

\section{Cyclin-Dependent Kinase 4 Correlates With Immune Regulation in Pancreatic Cancer}

Despite the importance of immune activation for cancer therapy (Sanmamed and Chen, 2018), experimental models for PDA therapeutics often fail to recapitulate host defense systems. Chou et al. (2018) used patient-derived tumor xenografts, which must be implanted into immunodeficient mice, to analyze the therapeutic efficacy of CDK4 inhibition on pancreatic cancer. This inevitably bypasses any immune involvement and fails to recapitulate the tumor immune microenvironment. Moreover, previous studies have validated the role of CDK4 in the regulation of cancer immunity and immunotherapy. Goel et al. (2017) reported that CDK4/6 inhibition triggers anticancer immune responses in breast cancer, and Zhang et al. (2018) showed that cyclin D-CDK4 kinase destabilizes programmed death-ligand 1 via the $\mathrm{Cul} 3^{\mathrm{SPOP}}$ complex to control cancer immunosurveillance in melanoma cancer. To determine the immunologic relevance of CDK4 in pancreatic cancer, we integrated all relevant datasets in the TCGA database for bioinformatics analyses. In pancreatic adenocarcinoma (PAAD), the results showed a positive correlation between CDK4 and the immunomodulatory system, including the immune effector molecules PRF1, GZMB, and IFNG (Figures 1A-C), as well as immune cells (naïve $\mathrm{T}$ cells, effector $\mathrm{T}$ cells, Th1like cells, central memory $\mathrm{T}$ cells, effector memory $\mathrm{T}$ cells, and resident memory $\mathrm{T}$ cells) (Figures 1D-I). These data suggest that the outcomes of CDK4-based PDA therapy in immunocompromised models have limited significance to the clinical treatment of PDA.

\section{Opposite Prognoses of Cyclin-Dependent Kinase 4 and 6 in Pancreatic Cancer}

Chou et al. (2018) recently highlighted the potential of CDK4 inhibitors as a targeted therapy for pancreatic cancer. However, Palbociclib (PD-0332991, Pfizer), the major CDK4-targeted drug used in the study, is a small molecule inhibitor designed to block the activity of both CDK4 and CDK6 to promote cytostasis (O'Leary et al., 2016). The concomitant effects of this therapy on CDK6 in more relevant physiological models of pancreatic cancer can, therefore, not be discounted. In light of this, we analyzed relevant genomic data and observed the opposite effects of CDK4 and CDK6 on pancreatic cancer prognosis. The expression levels of CDK4 and CDK6 were highly upregulated in PAAD compared with normal tissue (Figures 2A,B); however, CDK4 promoted rather than suppressed the OS of PDA patients (Figure 2C), whereas high CDK6 expression was associated with poor survival (Figure 2D). This phenotype implicates CDK6 as opposed to CDK4 for the poor prognosis of PDA in the clinic and questions the therapeutic benefits of Palbociclib in PDA therapy due to its synergistic effects on CDK4 and CDK6. In light of these data, patient-derived primary cell lines that genetically lack CDK4 and CDK6 should be generated to investigate their significance during Palbociclib treatment and exclude off-target effects. Of note, the combination of palbociclib and gemcitabine was investigated in the study as mentioned earlier, but the therapeutic efficacy of gemcitabine has been reported to be influenced by tumor immune microenvironment during pancreatic cancer therapy (Halbrook et al., 2019), which further highlights the importance of appropriate models in evaluating the CDK4/6-targeted combinational strategy.

\section{Retinoblastoma 1 Is Coupled to Cyclin-Dependent Kinase 4 and Poor Prognosis in Pancreatic Cancer}

Cyclin-dependent kinase $4 / 6$ controls the downstream transcriptional signals that regulate cancer cell proliferation through the phosphorylation of their canonical substrate RB1. CDK4/6-phosphorylated RB1 has been shown to promote anticancer immunity by inhibiting nuclear factor- $\kappa \mathrm{B}$ activation and programmed death-ligand 1 expression (Jin et al., 2019), further indicating the immunological relevance of palbociclib during cancer treatment. Moreover, aberrant CDK4/6-RB1 signaling has been reported in a range of cancers. Such alterations regulate immune evasion and immunotherapy (Hutcheson et al., 2015). Genomic analyses indicated that a similar phenotype exists in pancreatic cancer in which CDK4 regulates RB1 activation (Figures 3A,B). However, Chou et al. (2018) used only 19 whole-genome-sequenced pancreatic cancer patient-derived primary cell lines to exclude the potential impact of RB1 mutations in vivo. Although RB1 is highly expressed (Figure 3C) and positively related to CDK4 (Figure 3D) in PAAD, higher expression of RB1 was related to poor prognosis (Figure 3E), contradictory to the previous conclusions. The differential outcomes between genomic analysis and immunohistochemistry staining were caused by the failure to account for immune function and/or alterations in RB1. Hence, the phosphorylation of RB1 as a biomarker when predicting the therapeutic sensitivity of CDK4/6 inhibition requires further clinical and genetic analysis.

\section{CONCLUSION}

In summary, we highlight the importance of CDK4 for immune regulation. We further show that elevated CDK4 expression is a favorable prognostic index for cancer patients instead of high CDK6 expression that is close to poor prognosis. RB1 was positively related to CDK4 but linked to a poorer prognosis. These data suggest that the direct anti-tumor effects of CDK4/6 inhibition in xenografts fail to reflect their efficacy in pancreatic cancer patients. The utility of targeting CDK4/6 as a strategy for targeted-immunotherapy in pancreatic cancer now warrants further exploration. 


\section{DATA AVAILABILITY STATEMENT}

The original contributions presented in the study are included in the article/supplementary material, further inquiries can be directed to the corresponding author.

\section{AUTHOR CONTRIBUTIONS}

$\mathrm{XH}$ conceived this manuscript, conducted the analyses, and interpreted the data. $\mathrm{XH}$ and GZ drafted, discussed, and revised the manuscript. Both authors read and approved the final version.

\section{FUNDING}

This work was supported by grants from the National Natural Science Foundation of China (31970696 and 81502975 to XH).

\section{REFERENCES}

Chou, A., Froio, D., Nagrial, A. M., Parkin, A., Murphy, K. J., Chin, V. T., et al. (2018). Tailored first-line and second-line CDK4-targeting treatment combinations in mouse models of pancreatic cancer. Gut 67, 2142-2155. doi: 10.1136/gutjnl-2017-315144

Collisson, E. A., Bailey, P., Chang, D. K., Biankin, A. V., et al. (2019). Molecular subtypes of pancreatic cancer. Nat. Rev. Gastroenterol. Hepatol. 16, 207-220.

Goel, S., DeCristo, M. J., Watt, A. C., BrinJones, H., Sceneay, J., Li, B. B., et al. (2017). CDK4/6 inhibition triggers anti-tumour immunity. Nature 548, 471475. doi: 10.1038/nature23465

Halbrook, C. J., Pontious, C., Kovalenko, I., Lapienyte, L., Dreyer, S., Lee, H. J., et al. (2019). Macrophage-released pyrimidines inhibit gemcitabine therapy in pancreatic cancer. Cell Metab. 29, 1390.e6-1399.e6.

Hutcheson, J., Witkiewicz, A. K., and Knudsen, E. S. (2015). The RB tumor suppressor at the intersection of proliferation and immunity: relevance to disease immune evasion and immunotherapy. Cell Cycle 14, 3812-3819. doi: 10.1080/15384101.2015.1010922

Jin, X., Ding, D., Yan, Y., Li, H., Wang, B., Ma, L., et al. (2019). Phosphorylated RB promotes cancer immunity by inhibiting NF-kappaB activation and PD-L1 expression. Mol. Cell 73, 22.e6-35.e6.

Kleeff, J., Korc, M., Apte, M., La Vecchia, C., Johnson, C. D., Biankin, A. V., et al. (2016). Pancreatic cancer. Nat. Rev. Dis. Primers 2:16022.
The detailed process, including the study design, data analysis, and interpretation, as well as report writing, was not affected by the sponsored foundation.

\section{ACKNOWLEDGMENTS}

The authors would like to gratefully thank the TCGA (http://cancergenome.nih.gov) and other open-access cancer genome databases for data collection, and cBioPortal for Cancer Genomics (http://www.cbioportal.org) and Gene Expression Profiling Interactive Analysis 2 (http://gepia2.cancer-pku.cn) for providing data processing and customizable functions. Especially, XH would like to express deepest thanks to Prof. Guido Kroemer for the cancer immunity-associated technological training in his lab.

O'Leary, B., Finn, R. S., and Turner, N. C. (2016). Treating cancer with selective CDK4/6 inhibitors. Nat. Rev. Clin. Oncol. 13, 417-430. doi: 10.1038/nrclinonc. 2016.26

Rubin, S. M. (2013). Deciphering the retinoblastoma protein phosphorylation code. Trends Biochem. Sci. 38, 12-19. doi: 10.1016/j.tibs.2012.10.007

Sanmamed, M. F., and Chen, L. (2018). A paradigm shift in cancer immunotherapy: from enhancement to normalization. Cell 175, 313-326. doi: 10.1016/j.cell.2018.09.035

van den Heuvel, S., and Dyson, N. J. (2008). Conserved functions of the pRB and E2F families. Nat. Rev. Mol. Cell. Biol. 9, 713-724. doi: 10.1038/nrm2469

Zhang, J., Bu, X., Wang, H., Zhu, Y., Geng, Y., Nihira, N. T., et al. (2018). Cyclin D-CDK4 kinase destabilizes PD-L1 via cullin 3-SPOP to control cancer immune surveillance. Nature 553, 91-95. doi: 10.1038/nature25015

Conflict of Interest: The authors declare that the research was conducted in the absence of any commercial or financial relationships that could be construed as a potential conflict of interest.

Copyright (c) 2020 Huang and Zhang. This is an open-access article distributed under the terms of the Creative Commons Attribution License (CC BY). The use, distribution or reproduction in other forums is permitted, provided the original author(s) and the copyright owner(s) are credited and that the original publication in this journal is cited, in accordance with accepted academic practice. No use, distribution or reproduction is permitted which does not comply with these terms. 